\title{
Egypte: Encourager les journalistes à couvrir les questions relatives à la santé de la reproduction
}

Frontiers in Reproductive Health

Follow this and additional works at: https://knowledgecommons.popcouncil.org/departments_sbsr-rh

Part of the Journalism Studies Commons, Public Health Education and Promotion Commons, and the Technical and Professional Writing Commons

How does access to this work benefit you? Let us know!

\section{Recommended Citation}

"Egypte: Encourager les journalistes à couvrir les questions relatives à la santé de la reproduction," FRONTIERES Résumés de Recherche Opérationnelle. Dakar: Population Council, 2000. 


\title{
Egypte \\ Dissémination \\ $N^{\circ} 15$ \\ Encourager les Journalistes à couvrir les questions relatives à la Santé de la Reproduction
}

\begin{abstract}
Après avoir assisté à une série de réunions d'information sur les questions relatives à la santé de la reproduction, les journalistes de la presse écrite en Egypte ont amélioré leurs reportages sur ces questions. Les services de Santé peuvent améliorer les reportages sur les questions relatives à la Santé de la Reproduction en fournissant régulièrement des informations précises à un large éventail de journalistes.
\end{abstract}

\section{Contexte}

Afin de sensibiliser davantage le public sur les questions relatives à la Santé de la Reproduction (SR), le projet FRONTIERS du Population Council et le projet Policy Futures Group ont entrepris conjointement d'organiser des conférences de presse et de fournir aux grands journalistes des principaux journaux et revues des pays Arabes des documents de base. A cet effet, le personnel des dits projets a travaillé étroitement avec 20 journalistes Egyptiens parmi lesquels des rédacteurs en chef et des rédacteurs de la presse féminine, de Mai 1999 à Juin 2000. Les quatre conférences de presse ont porté sur les adolescents, les différents types de mariage, les procédés de contraception et la ménopause. Le dossier de presse préparé pour chaque conférence contenait des fiches d'informations, des documents de référence, une liste des adresses des principaux experts et une fiche d'évaluation.

Pour évaluer la couverture médiatique des sujets relatifs à la SR suite à l'intervention, le personnel du projet a effectué un suivi quotidien de 8 grands journaux et 9 magazines publiés en arabe. Tous les articles sur la SR ont été codés selon leur sujet, leur longueur et l'intégration des résultats issus des recherches.

\section{Résultats}

- Les conférences de presse ont été couvertes par les média. Sur les 433 articles publiés par les journaux sur la SR de Mai 1999 à Mars 2000, un cinquième traitait des sujets abordés lors des conférences de presse. Aussi, un tiers des 127 articles publiés dans les magazines sélectionnés couvraient des sujets abordés lors des conférences de presse.

\section{« Les chiffres et les schémas présentés.... auront plus d'impact sur l'opinion publique»}

(Propos d'un journaliste participant)

Les journalistes ayant assisté aux conférences de presse disent qu'ils ont acquis plus de connaissances sur les questions relatives à la SR et qu'ils projettent d'utiliser les dossiers de presse pour écrire leurs articles. Certains journalistes ont partagé leurs dossiers avec leurs collègues.

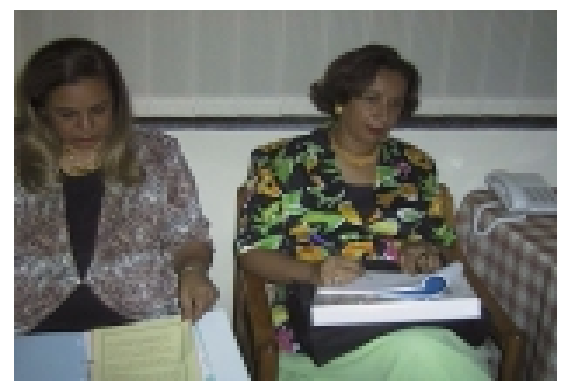


- Le projet a certes amélioré la qualité des reportages, mais beaucoup de travail reste à faire. Presque le tiers des articles sur les conférences de presse ont cité des résultats de la recherche ou des informations contenues dans le dossier de presse. Malgré le fait que le personnel du projet insistait sur la nécessité de varier les sources d'informations, la plupart des articles s'étaient limités sur une seule source d'informations.

Sur le nombre total des pages réservées à la $\mathrm{SR}$, les questions liées à la maternité sans risque sont traitées sur plus d'une page sur quatre.

\section{Sujets relatifs à la SR couverts par les journaux et les magazines Nombre de pages publiées}

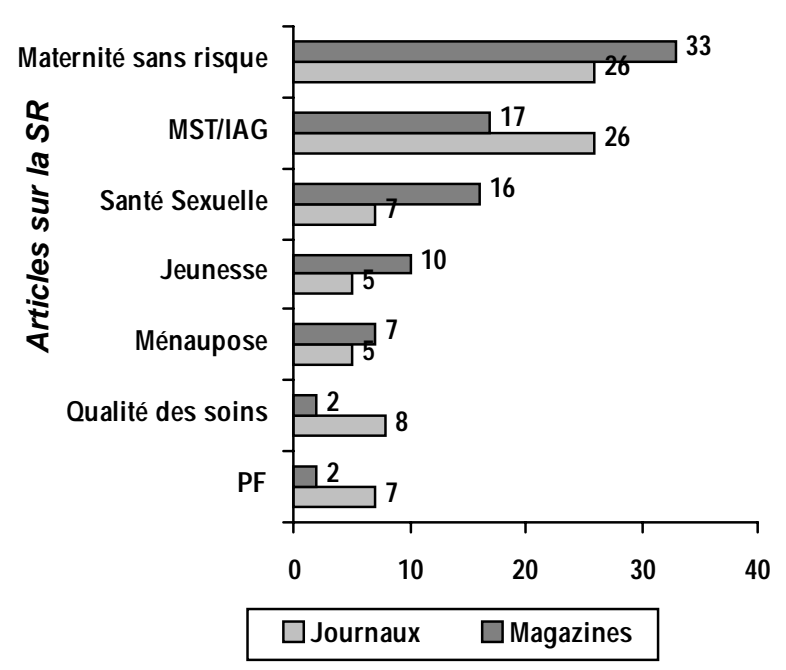

Les infections de l'appareil génital figuraient en deuxième position, suivies de la santé sexuelle, des adolescents, de la ménopause et de la qualité des soins. (voir schéma).
- La majorité des articles sur la SR parus dans les journaux traitaient des faits d'actualité. En revanche, plus de la moitié, parus dans les magazines, était des articles de fond ; la moitié de ceux-ci faisaient une page ou plus. Ces deux supports de publication permettent de disséminer la recherche : les journaux sont largement lus par les décideurs politiques qui, par contre, ont tendance à garder les magazines plus longtemps et à les partager avec les autres.

\section{Implications pour les programmes}

- La dissémination des résultats des recherches doit nécessairement inclure des réunions d'information et de la documentation pour les journalistes. Des liens étroits avec la presse écrite et parlée doivent être développés.

Il faut impliquer un plus grand nombre d'agences nationales dans les campagnes médiatiques pour permettre une couverture plus variée des sujets relatifs à la SR. Etant donné la faible couverture médiatique actuelle des sujets relatifs à la Planification familiale et à l'excision*, on doit mettre plus d'informations relatives à ces sujets à la disposition des journalistes.

Les Services de Santé doivent prendre les dispositions nécessaires pour améliorer la qualité des reportages en fournissant régulièrement aux journalistes des informations précises et en les aidant à identifier des sujet plus captivants.

\section{* Voir OR Résumé 14, Les ONG doivent unir leurs forces pour éradiquer l'excision}

Hegazi, Shar and Mona Khalifa. 2000. « Accroître la Couvertures des questions relatives à la Santé de la Reproduction dans la Presse Egyptienne : Rapport Final ». Pour de plus amples informations ou pour obtenir une copie de la version Anglaise du Rapport de cette étude ; contacter : Population Council, 6A Giza St., P.O. Box 115, Dokki 12211, Giza, Cairo, Egypte. Tél. : 202-5725910 ; Fax : 202-5701804 ; E-mail : frontiers@pccairo.org.

Ce projet a été effectué avec l'appui de l'AGENCE AMERICAINE POUR LE DEVELOPPEMENT INTERNATIONAL (USAID) sous l'Accord de Coopération Numéro HRN-A-00-98-00012-00.

\section{(1) Population Cound}

